\title{
Serum Neurofilament Light Chain Measurement in MS: Hurdles to Clinical Translation
}

\author{
Simon Thebault ${ }^{1 *}$, Ronald A. Booth ${ }^{2}$, Carolina A. Rush ${ }^{1}$, Heather MacLean ${ }^{1}$ and \\ Mark S. Freedman ${ }^{1 *}$ \\ ${ }^{1}$ Department of Medicine, The Ottawa Hospital Research Institute, The University of Ottawa, Ottawa, ON, Canada, \\ ${ }^{2}$ Department of Pathology and Laboratory Medicine, The Eastern Ontario Regional Laboratory Association, The Ottawa \\ Hospital, Ottawa Hospital Research Institute, The University of Ottawa, Ottawa, ON, Canada
}

\section{OPEN ACCESS}

Edited by:

Isabella Zanella,

University of Brescia, Italy

Reviewed by:

Tomas Uher,

Charles University, Czechia

Özgür Yaldizli,

University Hospital of Basel,

Switzerland

Ching-Hua Lu,

China Medical University, Taiwan

*Correspondence:

Simon Thebault

sthebault@toh.ca;

thesdx@gmail.com

Mark S. Freedman

mfreedman@toh.ca

Specialty section:

This article was submitted to

Neurodegeneration,

a section of the journal

Frontiers in Neuroscience

Received: 18 January 2021

Accepted: 08 March 2021

Published: 25 March 2021

Citation:

Thebault S, Booth RA, Rush CA,

Maclean H and Freedman MS (2021)

Serum Neurofilament Light Chain

Measurement in MS: Hurdles

to Clinical Translation.

Front. Neurosci. 15:654942.

doi: 10.3389/fnins.2021.654942
Measurement of serum neurofilament light chain concentration (sNfL) promises to become a convenient, cost effective and meaningful adjunct for multiple sclerosis (MS) prognostication as well as monitoring disease activity in response to treatment. Despite the remarkable progress and an ever-increasing literature supporting the potential role of sNfL in MS over the last 5 years, a number of hurdles remain before this test can be integrated into routine clinical practice. In this review we highlight these hurdles, broadly classified by concerns relating to clinical validity and analytical validity. After setting out an aspirational roadmap as to how many of these issues can be overcome, we conclude by sharing our vision of the current and future role of sNfL assays in MS clinical practice.

Keywords: multiple sclerosis, translation, neurofilament light, blood, biomarker

\section{INTRODUCTION}

The spectrum of multiple sclerosis (MS) disease severity is broad, encompassing mild or even benign forms of the disease that may not require treatment at all (Sartori et al., 2017) to rapid progressors who accumulate irreversible worsening early-on unless drastic interventions are made (Atkins et al., 2016). Due to advancements in disease modifying therapies (DMT) for MS over the past two decades, a range of treatment options are now available, and "no evidence of disease activity" on clinical and MRI measures is a realistic treatment goal for many patients (Lublin, 2012). Following assessment of demographic, clinical and MRI features, patients identified as having more severe disease are increasingly treated with higher efficacy therapies up-front, with a lower threshold for treatment escalation upon disease breakthrough on less effective treatments (Giovannoni, 2018). However, a double-edged sword, the expanding range of higher efficacy immunosuppressive therapies is often accompanied by toxicity and iatrogenic morbidity. As such, neurologists aspire to closely titrate the minimal treatment intensity required to achieve disease control, and quickly react to breakthrough activity thereafter (Freedman et al., 2020). However, the current status quo of disease prognostication and monitoring in MS in routine clinical practice leaves much to be desired, lagging behind the therapeutic advancements. Clinical decisionmaking is still dependent on a synthesis of incomplete clinical and MRI information. Initial treatment selection remains a vaguely informed decision, based on our best assessment and patient preferences, but conflated by financial/insurance considerations and clinician preference. Once on a given therapy, subsequent escalation of therapy often lags behind the damaging disease activity that neurologists and their patients seek to prevent. Fluid biomarkers that conveniently and accurately 
measure and track subclinical disease activity have been long sought to fill this knowledge and practice gap (Comabella and Montalban, 2014). After decades of searching, serum concentrations of neurofilament light chain (sNfL) have emerged over the past few years as a promising candidate.

Neurofilament light chain (NfL) is the most abundant of a family of highly conserved neuron-specific structural neurofilament proteins (Fuchs and Cleveland, 1998). Although our understanding of the physiology, pathophysiology and kinetics remains incomplete, it has been known for some time using conventional assays that CSF NfL is elevated in neurological conditions that cause neuroaxonal damage, such as MS (Eikelenboom et al., 2003). In the last 5 years, with the advent of ultrasensitive single molecule detection technologies, reliable blood measurements which correlate with CSF concentrations has become possible (Kuhle et al., 2016a; Wilson et al., 2016). Although concentrations in serum and plasma are closely related, they are not interchangeable; as the majority of current evidence relates to NfL concentrations in serum (sNfL), this is the focus of this review. Analogous to the cardiologist's troponin, the promise of a serum test of neuroaxonal damage has driven remarkable interest (Thebault et al., 2020b). Although sNfL has broader applicability, MS has become the test case for sNfL clinical utility due the unmet need for biomarkers and facilitated by extensive availability of specimens from retrospective cohorts (Thebault et al., 2021). In MS, sNfL is primarily a surrogates of inflammatory disease activity, the highest concentrations seen around the time of relapses and new MRI lesions, where sNfL trends up and then down for several months before and after (Kuhle et al., 2016b; Akgün et al., 2019). In pragmatic clinical settings outside of clinical trials, sNfL decreases broadly in line with demonstrated treatment efficacies (Bittner et al., 2020; Delcoigne et al., 2020). Furthermore, higher sNfL is predictive of poorer future clinical outcomes at every stage. NfL concentrations are elevated 6 years prior to the clinical onset of MS (Bjornevik et al., 2020). In clinically isolated syndromes, higher sNfL independently predicted faster conversion to clinically definite MS (Disanto et al., 2016). Following diagnosis, higher sNfL has been associated with short and long term poorer outcomes, including relapses, EDSS score progression including progression independent of relapse-activity, clinical conversion to a progressive phenotype, poorer cognitive measures as well as both MRI lesion activity and atrophy (Disanto et al., 2017; Barro et al., 2018; Chitnis et al., 2018; Siller et al., 2018; Cantó et al., 2019; Lorscheider and Benkert, 2020; Thebault et al., 2020a). Some experts consider this test to be on the cusp of widespread clinical adoption (Leppert and Kuhle, 2019), while others remain skeptical (Javed and Stankiewicz, 2020). As early adopters of sNfL testing in MS, in this review, we summarize what we see are key unknowns before the test could and should be deployed in routine clinical practice.

\section{Hurdles to Widespread Clinical Translation of SNfL}

Despite showing great promise, there are a number of issues relating to sNfL that must be either overcome or at least better appreciated before it can be considered part of the routine armamentarium of MS care (Figure 1). Challenges can be summarized in terms of analytical validity (test performance for sNfL) and clinical validity (sNfL performance as a surrogate of MS-related clinical outcomes of interest). Regulatory approvals of the test are contingent on both components being satisfactorily being met.

\section{CLINICAL VALIDITY}

sNfL is not specific for MS pathology. While there is an elegance to this, as both inflammatory and neurodegenerative activity in MS are summarized in a single marker, this means sNfL is not a diagnostic marker in MS. Unlike MRI, where new lesions have a characteristic appearance and can be specially correlated with clinical signs and symptoms, sNfL is agnostic of the underlying pathological process causing neuronal loss. Thus, if an individual patient's concentration is found to be high or rising, neurologists need to carefully consider confounders such as age and comorbidities (discussed in detail below) and may need to order additional testing such as MRI to clarify the situation.

Furthermore, there is significant overlap in sNfL in MS patients and healthy controls, even including cohorts of patients with the most aggressive forms of MS (Thebault et al., 2019). Owing to the relapsing remitting nature of the condition clinically in many, at any given time, the majority of MS patients might be expected to have similar sNfL to healthy controls (Barro et al., 2018). Concentrations frequently fall in an intermediate/gray zone. Thus, while the correlation between sNfL and important MS outcomes in cross-sectional studies is remarkable, interpretation of individual sNfL concentrations in clinical decision making remains challenging. This again highlights the need for careful consideration or even adjustment for the principal clinical confounders.

\section{Age}

Age is the principal physiologic determinant of sNfL. This is likely attributable to the cumulative effects of subclinical pathologies, such as white matter disease causing accelerated neuronal loss (Khalil et al., 2020). There is a moderate association between sNfL and age in both healthy controls and MS patients alike, with typical $r$ values ranging from 0.6 to 0.7 (Khalil et al., 2020) and an increase in adult control sNfL levels of $2.2 \%$ per yer of age (Disanto et al., 2017; Barro et al., 2018). In healthy controls, there is an inflection around the age of 60 , after which subsequent age-related sNfL increases accelerate, as does inter-individual variability within a given age cohort (Khalil et al., 2020).

There are two possible solutions to this problem. Firstly, patients could serve as their own baseline, using concentrations obtained during a stable period of remission as the comparator for subsequent serial measurements. This could not only account for age but also other commodities which we outline in the sections below. Using such a technique in a prospective observational cohort of 15 MS patients sampled during alemtuzumab treatment, one study found that sNfL "peaks" (>3 standard deviations above steady state concentrations) were 


\section{Clinical validity}

Non-specifity to MS
Overlap with control levels
Confounders: Age, comorbidities
Relation to lesion location?
Plasma levels lower than serum
Limited understanding of
kinetics/ dynamics

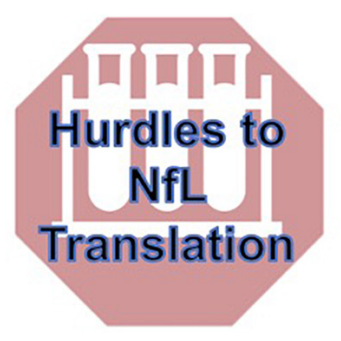

2. Analytical validity

Preanalytical considerations

Utility, reliability, repeatability

Assay standardization

Analytical standardization

3. Regulatory approvals

FIGURE 1 | Barriers to the clinical translation of sNfL in 2021.

associated with clinical and MRI activity in the majority of cases (Akgün et al., 2019). The downside to this approach is that it requires baseline measurement(s) during a preceding period of stability to serve as a subsequent longitudinal benchmark: this is contentious to define and difficult or impossible to obtain early on in the most active patients who would benefit the most from close monitoring. In this situation, a lack of reduction in sNfL following treatment initiation is itself meaningful (Huss et al., 2020) and could be used to guide escalation.

Alternatively, or perhaps in conjunction, others have adjusted for age by comparison to normative datasets from healthy controls. This approach is principally limited by the availability of large biobanks of healthy control sera required to generate such data. The Swiss group based in Basel has been particularly successful, initially presenting patient data in relation to percentiles of healthy control concentrations (Barro et al., 2018), but more recently and statistically rigorously as $z$-scores of lognormalized sNfL (Yaldizli et al., 2020). The availability of such normative datasets as well as ability of local laboratories to apply an age-adjustment factor is undetermined. Nonetheless, a relatively simple age adjustment which can be calculated for both a single measurement as well as serial measurement means is a significant step toward being able to use sNfL measurement to follow individual patents.

\section{Confounding Effects of Other Neurological and Non-neurological Comorbidities}

Extensively reviewed elsewhere (Khalil et al., 2018; Barro et al., 2020), higher sNfL is seen in many central and peripheral nervous system diseases that involve neuroaxonal injury including neurodegenerative conditions (Forgrave et al., 2019), stroke (Nielsen et al., 2020- plasma concentrations), and peripheral neuropathies (Altmann et al., 2020). Analogous to troponin in cardiac disease, clinical context is required. Fortunately, many alternate explanations of an elevated sNfL are usually clinically apparent or uncommon in the demographic of MS patients requiring active surveillance. More troubling however is the increase in sNfL seen following even mild traumatic brain injury. Here concentrations increase acutely, are predictive of the severity of injury, and remain elevated for several years after the injury (Shahim et al., 2020). High risk groups include military personnel (Boutté et al., 2019) and athletes (Shahim et al., 2018plasma concentrations). In the context of MS and superimposed head injury, it may be difficult to attribute concentrations or dynamic changes to one pathology or the other.

Iatrogenic causes of sNfL elevation have also been identified. In a cohort of patients over 60 years old serially sampled after non-neurological surgeries requiring general anesthesia (mostly arthroscopies), concentrations increased by $67 \%$ and remained elevated beyond $48 \mathrm{~h}$ (Evered et al., 2018). An important consideration for MS patients is the possible effects of lumbar puncture: in Macaque monkeys, a lumbar puncture in the preceding 2-3 weeks increased median sNfL by $162 \%$ (Boehnke et al., 2020). Although lumbar punctures are generally infrequent events for most MS patients, much of our current understanding of sNfL is derived from intensively investigated cohorts of patients undergoing treatments, many of which underwent frequent lumbar puncture, a possible confounder of concern. Thus, appropriate timing of blood collection is exceedingly important for correct interpretation and can mitigate the potential for misinterpretation of results.

MS patients can also be at risk for other neurological complications that cause sNfL concentrations to rise. For instance, a 10-fold increase was noted at the time of onset of natalizumab-induced progressive multifocal leukoencephalopathy (Dalla Costa et al., 2019). In a cohort of patients undergoing ablative hemopoietic stem cell transplantation for aggressive MS, transient increases in the first year after the treatment reflected chemotherapeutic toxicity (Thebault et al., 2020c). Nonetheless, we feel that the identification of a rapidly rising NfL in these situations could be a useful warning signal to trigger a reassessment and additional investigations to identify the cause, or switch therapy.

Non-neurological conditions are also known to affect sNfL and need to be considered in any comorbid patient. BMI has been shown to have an effect on the sNfL, likely due to an increase in volume of distribution, where every $1 \mathrm{~kg} / \mathrm{m}^{2}$ rise in body mass index, sNfL decreases by $0.02 \mathrm{pg} / \mathrm{ml}$ (Manouchehrinia et al., 2020). Data from the stroke literature suggests that cardiovascular 
risk factors including hypertension and poor glycemic control and perhaps renal function are also associated with higher sNfL (Korley et al., 2019). Similar to the proposed explanations for increased concentrations with age, these associations could be driven by comorbid but clinically silent white matter disease (Khalil et al., 2020). Renal function may also be important for NfL clearance as a cause for higher measurements in these patients (Akamine et al., 2020).

\section{Other Physiologic Considerations}

Our understanding of the pathophysiologic processes surrounding NfL release, distribution and metabolism are incomplete. This is illustrated by the correlation of serum and CSF concentrations, which typically has an $r$-value of $0.7-0.8$ (e.g., Thebault et al., 2019). This equates to about $50 \%$ of sNfL being directly attributable to CSF NfL concentrations. Once NfL leaves axons and enters the extracellular space, it is not known what proportion is drained by lymphatic routes vs. direct drainage into CSF. Both individual and dynamic differences in these routes vary in a manner that could impact sNfL. In the CSF, there could be regional variation in NfL correlation, for example in the cul-de-sac of the lumbar cistern where CSF sampling occurs. Blood brain barrier permeability itself may be a confounder; NfL quotient in serum compared to CSF could be selectively increased following periods of inflammation such as that seen in MS relapse, positively skewing serum measures. However recent studies on this topic in MS patients present conflicting results (Kalm et al., 2017; Engel et al., 2020; Uher et al., 2020a). The diurnal timing of blood collection may also be an important consideration; in a study of 15 healthy males, one group found a more than $10 \%$ increase in plasma concentrations of NfL in the morning compared to the evening, although were surprised to find that elevation was not seen following acute sleep deprivation (Benedict et al., 2020). A hypothetical explanation for this diurnal variation proposed by the authors is that synaptic pruning in sleep may alter NfL kinetics.

Once NfL enters the blood, there are other physiologic considerations. One such possible confounder is existence of anti-NfL antibodies found in many MS patients (Silber et al., 2002). While the pathogenic potential of these antibodies is debatable, the presence or absence of these antibodies could alter peripheral NfL clearance.

Related to the physiologic kinetics of NfL distribution and clearance, the half-life of $\mathrm{sNfL}$ is a key consideration with implications on the frequency of disease activity monitoring. In a longitudinal study of NfL before and after intrathecal catheter insertion, NfL in both CSF and serum peaked at 1 month postsurgery, returning to baseline after 6-9 months (Bergman et al., 2016). In longitudinally sampled MS patients around the time of relapse, sNfL increased 5 months before, peaked at clinical onset, and recovered within 4-5 months (Akgün et al., 2019). In another observational cohort of 94 patients enrolled in the Comprehensive Longitudinal Investigation of Multiple Sclerosis at the Brigham and Women's Hospital (CLIMB) study, sNfL was elevated by one third in a 3 month window around gadolinium (Gd) enhancing lesions compared to remission samples (Rosso et al., 2020). Thus, while some possible individual influences of sNfL kinetics remain ill-defined, many groups are now selecting testing frequencies in the 3-6 month range for MS disease activity monitoring.

\section{Possible Importance of MS Lesion Location}

It is the authors opinion that lesion location may be an important consideration in the interpretation of sNfL. To date, all studies have compared sNfL concentrations to total whole brain lesion volumes on MRI, and identified this to be one of the most consistent associations of sNfL. However, a large lesion in the right frontal lobe would likely result in a very significant elevation in sNfL conceivably with minimal appreciable disability. Conversely a small lesion affecting key brainstem structures may result in a smaller sNfL rise but significant long-term disability. Additionally, we speculate that other factors such as axon density in different brain and spine regions could be important determinants of the quantitively rise in $\mathrm{NNfL}$ in response to a given lesion.

\section{ANALYTICAL VALIDITY}

\section{Preanalytical Considerations}

Variations in sample acquisition, transport, processing and storage prior to protein quantification are important preanalytical confounders for many blood biomarkers. Although serum and plasma neurofilament levels are very strongly correlated ( $r=0.96$, Sejbaek et al., 2019), plasma concentrations are around $25 \%$ lower than paired serum concentrations, highlighting the need to standardize blood measurements to a single specimen type. For this reason in this review we have chosen to focus on serum as the more prevalent and studied blood biofluid to promote comparability and utility. Otherwise, sNfL has shown good stability over multiple freeze-thaw cycles and prolonged exposure to room temperature (e.g., Hviid et al., 2020, reviewed by Table 1 in Barro et al., 2020).

\section{Assay Standardization}

Much of the focus on sNfL in recent years is directly attributable to development of a clinical immunoassay platform capable of detecting the low concentrations in blood. The Single Molecule Array (SiMoA) has transformed NfL from a CSF-only research-marker of merit to its current status on the verge of clinical translation in blood (Kuhle et al., 2016a). Comparison of traditional ELISA with electrochemiluminescence and SiMoA demonstrated the superiority of SiMoA with an analytical sensitivity of $0.62 \mathrm{pg} / \mathrm{mL}$ compared with $15.6 \mathrm{pg} / \mathrm{mL}$ electrochemiluminescence and $78.0 \mathrm{pg} / \mathrm{mL}$ for enzyme linked immunosorbent assay (Kuhle et al., 2016a). This increased sensitivity of SiMoA is able to detect sNfL in $100 \%$ of healthy individuals. The SiMoA assay uses a unique ELISA method of detecting very low concentration analytes (Rissin et al., 2010). Briefly, antibodies are linked to a solid surface as in a traditional ELISA, however the SiMoA assay utilizes microbeads 
2.7 $\mu \mathrm{m}$ in size that individually fit into a microwell array. When measuring very low concentration analytes (subfemtomolar concentrations), the antigen-bead ratio is approximately 1:1 and follow a Poisson distribution. This distribution suggests that beads carry either a single immunocomplex or none, and with very low analyte concentrations only $1-2 \%$ of beads carry an immunocomplex. Detection of such low concentrations is not possible through routine enzymatic methods. To accomplish detection, each individual bead is loaded into a single microwell which can be "digitally" counted. Detection is through fluorescent labeling of immunocomplexes which is sensitive enough to measure a single immunocomplex on a single bead. In this way, the number of beads are counted and quantitated against a standard curve, allowing extremely low analyte concentrations to be reliably measured.

Use of the SiMoA assay has facilitated the measurement of NfL in blood and allowed much of the research in MS. The initial NfL assay developed for the SiMoA assay used a home-brew method developed by the Basel group (Kuhle et al., 2016a). They used monoclonal NfL antibodies developed by Umam Diagnostics (47:3 and 2:1, subsequently purchased by Quanterix) along with bovine NfL calibrators. The majority of the early studies were completed using the home-brew assay. More recent studies use the commercially available Quanterix NF-light ${ }^{\mathrm{TM}}$ assay kit which uses recombinant human (rhuman) NfL calibrators. It is important for investigators and clinicians to recognize which assay has been used, as that there is a significant positive bias $(5: 1)$ of the home-brew assay relative to the commercial NF-light ${ }^{\mathrm{TM}}$ assay (Hendricks et al., 2019).

These assay differences highlights the need for assay standardization, and the role of multi-site validation to inform reproducibility and create standardization protocols. In one such international validation effort of the Quanterix NF-light ${ }^{\mathrm{TM}}$ assay that sought to assess a variety of analytical outcomes including instrument qualification, precision, level of detection and level of quantification, parallelism and proficiency, the assay performed well across 17 sites with intra- and inter-assay coefficients varying less than 6 and 9\%, respectively (Kuhle et al., 2018). However, as interest in this biomarker increases, several other assay platforms are now showing near-equivalent dynamic range and level of detectability. While this competition will drive down setup and testing costs to increase availability, careful work will be needed to confirm inter-platform equivalence.

\section{Data Analysis and Clinical Reporting}

Of the published data available, there is significant variation in data analysis methodologies, limiting inter-study comparability or subsequent meta-analyses. Groups have variably reported measures of central tendency with the mean, median, and geomean. Subsequently reported statistics have included a mixture of parametric and non-parametric techniques, sometimes inappropriately deployed. In our experience, sNfL concentrations are distributed logarithmically. Therefore, comparisons of raw conentrations are constrained to nonparametric techniques, whereas more powerful parametric statistics are possible following $\mathrm{Ln} / \mathrm{log}$ transformation.
Preliminary data from the Basel group (Yaldizli et al., 2020) takes this one step further as they generated age-adjusted z-scores of log transformed data. Although use of $z$-scores may be the most appropriate technique for dealing with age-related increases in sNfL, it may pose challenges for reporting from clinical diagnostic laboratories. Similarly, future data analysis challenges are to determine the most meaningful and clinically deployable measure of sNfL change when trending values. Currently, it is unclear if the raw number alone, reported with reference to an age adjusted population, is important or if an absolute or relative increase is most clinically relevant. Regardless, with every statistical manipulation beyond simple reporting of raw values and cut-offs, may cause implementation hurdles in the clinical diagnostic laboratory and may become practically challenging in a real-world clinical setting.

\section{A CURRENT ROLE FOR sNfL IN MS?}

We already know that as an adjunctive measure in MS, high or increasing concentrations of sNfL are associated with relapses, EDSS worsening, lesions on MRI scans and atrophy of both the brain and spinal cord. Conversely, serially low sNfL is reassuring. Yet many see the limitations and unknowns relating to the precise interpretation of individual $\mathrm{sNfL}$ concentrations so problematic that the marker is "not ready for prime time" (Javed and Stankiewicz, 2020). However, it is the authors' opinion that the clinical translation of sNfL need not be so black or white. To demand stringent criteria for clinical translation not only ignores the rapidly accumulating body of evidence that already indicates utility for the marker but also seems like a double standard. Neurologists have long tried to use the accurate, often machine generated changes on serial MRI scanning in clinical trials to estimate disease change in their MS patients, only to be challenged with inaccuracies in real life (as opposed to carefully regimented clinical trial MRI studies) due to malalignment, different MRI sequences, different scanners or simply differences in the quality of imaging. Despite this, MRI has become the gold standard non-clinical means for measuring disease in MS. This has not stopped clinicians from making interpretations from serial scans to inform on treatment decisions.

It is our opinion that with a good appreciation of the shortcomings and pitfalls, individual patient $\mathrm{sNfL}$ concentrations are already a helpful adjunct to clinical practice. In Figure 2 we propose how sNfL can be incorporated into clinical practice as it currently stands: an imperfect marker, that should never be interpreted in isolation. We find it a helpful adjunctive tool and a useful trigger for expedited reassessment when unexpectedly high or rising. Better age dependent based on parametric $z$-score cutoffs (rather than non-parametric percentile cutoffs) are imminently and eagerly awaited. As concerns of clinical validity are better understood if not accounted for and the analytical validity is further established, we hope this marker will be further incorporated into the standard of care. Enabled by less constraining approval processes for clinical use in some jurisdictions, some centers such as our own are already using this test routinely in the MS clinic. 


\section{sNfL, A FUTURE STANDARD OF MS CARE?}

Analogous to the implementation of MRI in routine disease activity monitoring in MS, neurologists will require some education on how to correctly interpret sNfL and incorporate it into routine clinical practice. Similar to MRI, using sNfL may require the establishment of a "baseline" from whence future changes can be referenced and size changes be interpreted. The establishment of better of age-adjusted normative datasets (reference intervals) and biological variation (reference change values) will be a vital step in further individualizing sNfL group level associations. As with many new technologies, the cost of NfL testing itself remains high; competition will help reduce these costs but also present new issues relating to inter-assay comparability (Figure 3).

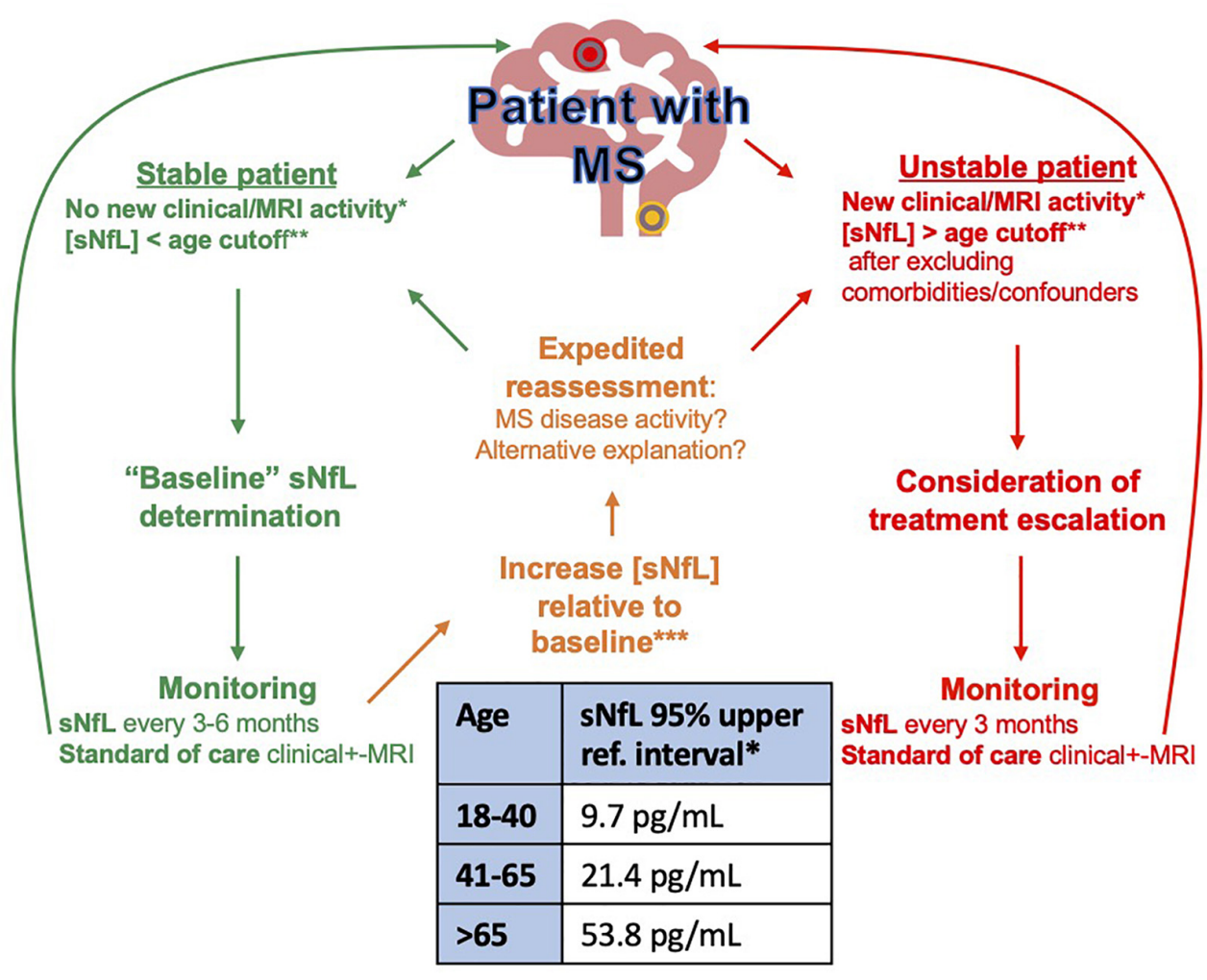

FIGURE 2 | Proposed algorithm for NfL monitoring in MS. ${ }^{*}$ Clinical or MRI disease activity: New relapses, EDSS worsening, New/enlarging MRI lesion. ${ }^{* *}$ sNfL 95\% age-dependent upper reference interval calculated on SiMOA HD1 instrument using Quanterix NF-light ${ }^{T M}$ (Hviid et al., 2020). ${ }^{* *}$ The increase in sNfL from baseline that best denotes impending disease activity that should prompt further action is still to be determined. Preliminary data from 58 patients with MS followed every 3 months over 1 year suggests that a doubling of sNfL from baseline is associated with a $2.2 \times$ relative risk of relapse (Thebault, Unpublished observation).

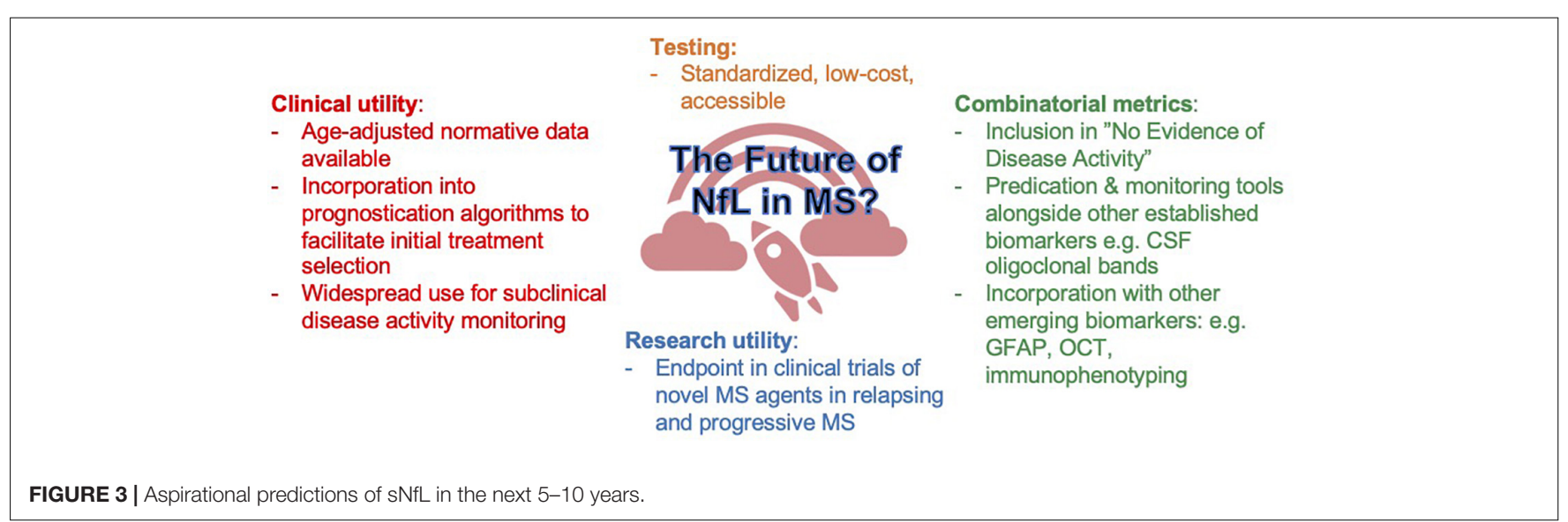


sNfL in patients with early-stage disease could be incorporated into prognostic models and aid initial treatment selection. Serial measurements, for instance every 3 months, could be useful to monitor for subclinical disease activity both on or off treatment. Here, increasing sNfL would be an objective trigger for neurologists to consider expedited clinical and MRI reassessment, and serially low or stable sNfL would be reassuring (Uher et al., 2020b). There is already substantial evidence for sNfL to be included in future definitions of "no evidence of disease activity." Thus, through more refined initial treatment selection and closer disease activity monitoring, we think sNfL could have the power to modify the trajectory of MS for the better and improve outcomes. Furthermore, sNfL could reduce current costs by optimizing utilization of MRI, where annual scans for all clinically stable patients is not only expensive but also unfeasible in many settings and could be perhaps better targeted to patients with high or rising

\section{REFERENCES}

Akamine, S., Marutani, N., Kanayama, D., Gotoh, S., Maruyama, R., Yanagida, K., et al. (2020). Renal function is associated with blood neurofilament light chain level in older adults. Sci. Rep. 10, 1-8. doi: 10.1038/s41598-020-76990-7

Akgün, K., Kretschmann, N., Haase, R., Proschmann, U., Kitzler, H. H., Reichmann, H., et al. (2019). Profiling individual clinical responses by highfrequency serum neurofilament assessment in MS. Neurol. Neuroimmunol. NeuroInflammation 6:e555. doi: 10.1212/NXI.0000000000000555

Altmann, P., De Simoni, D., Kaider, A., Ludwig, B., Rath, J., Leutmezer, F., et al. (2020). Increased serum neurofilament light chain concentration indicates poor outcome in Guillain-Barré syndrome. J Neuroinflammation 17:86.

Atkins, H. L., Bowman, M., Allan, D., Anstee, G., Arnold, D. L., Bar-Or, A., et al. (2016). Immunoablation and autologous haemopoietic stem-cell transplantation for aggressive multiple sclerosis: a multicentre single-group phase 2 trial. Lancet 388, 576-585. doi: 10.1016/S0140-6736(16)30169-6

Barro, C., Benkert, P., Disanto, G., Tsagkas, C., Amann, M., Naegelin, Y., et al. (2018). Serum neurofilament as a predictor of disease worsening and brain and spinal cord atrophy in multiple sclerosis. Brain 141, 2382-2391. doi: 10.1093/ brain/awy 154

Barro, C., Chitnis, T., and Weiner, H. L. (2020). Blood neurofilament light: a critical review of its application to neurologic disease. Ann. Clin. Transl. Neurol. 7, 2508-2523. doi: 10.1002/acn3.51234

Benedict, C., Blennow, K., Zetterberg, H., and Cedernaes, J. (2020). Effects of acute sleep loss on diurnal plasma dynamics of CNS health biomarkers in young men. Neurology 94, e1181-e1189. doi: 10.1212/WNL.0000000000008866

Bergman, J., Dring, A., Zetterberg, H., Blennow, K., Norgren, N., Gilthorpe, J., et al. (2016). Neurofilament light in CSF and serum is a sensitive marker for axonal white matter injury in MS. Neurol. Neuroimmunol. Neuroinflammation 3:e271. doi: 10.1212/nxi.0000000000000271

Bittner, S., Steffen, F., Uphaus, T., Muthuraman, M., Fleischer, V., Salmen, A., et al. (2020). Clinical implications of serum neurofilament in newly diagnosed MS patients: a longitudinal multicentre cohort study. EBioMedicine 56, 1-13. doi: 10.1016/j.ebiom.2020.102807

Bjornevik, K., Munger, K. L., Cortese, M., Barro, C., Healy, B. C., Niebuhr, D. W., et al. (2020). Serum neurofilament light chain levels in patients with presymptomatic multiple sclerosis. JAMA Neurol. 77, 58-64. doi: 10.1001/ jamaneurol.2019.3238

Boehnke, S. E., Robertson, E. L., Armitage-Brown, B., Wither, R. G., Lyra e Silva, N. M., Winterborn, A., et al. (2020). The effect of lumbar puncture on the neurodegeneration biomarker neurofilament light in macaque monkeys. Alzheimers Dement. Diagnosis, Assess. Dis. Monit. 12, 1-8. doi: 10.1002/dad2. 12069

Boutté, A. M., Thangavelu, B., LaValle, C. R., Nemes, J., Gilsdorf, J., Shear, D. A., et al. (2019). Brain-related proteins as serum biomarkers of acute, subconcussive blast overpressure exposure: a cohort study of military personnel. PLoS One 14:e0221036. doi: 10.1371/journal.pone.0221036
sNfL. While the role of sNfL as a clinically useful marker in progressive MS is less clear, this remains a key area of need where clinical responsiveness can be more difficult to quantify. Finally, the potential of sNfL may be augmented by the inclusion of additional markers into combinatorial metrics. While sNfL represents an important first step in a biomarkerdriven personalization of MS care, it certainly will not be the last.

\section{AUTHOR CONTRIBUTIONS}

ST conceived the study, performed the literature review drafted, and edited the manuscript. RB, CR, and HM drafted the manuscript. MF conceived the study, drafted, and edited the manuscript. All authors contributed to the article and approved the submitted version.

Cantó, E., Barro, C., Zhao, C., Caillier, S. J., Michalak, Z., Bove, R., et al. (2019). Association between serum neurofilament light chain levels and longterm disease course among patients with multiple sclerosis followed up for 12 years. JAMA Neurol. 76, 1359-1366. doi: 10.1001/jamaneurol.2019. 2137

Chitnis, T., Gonzalez, C., Healy, B. C., Saxena, S., Rosso, M., Barro, C., et al. (2018). Neurofilament light chain serum levels correlate with 10-year MRI outcomes in multiple sclerosis. Ann. Clin. Transl. Neurol. 5, 1478-1491. doi: 10.1002/acn3. 638

Comabella, M., and Montalban, X. (2014). Body fluid biomarkers in multiple sclerosis. Lancet Neurol. 13, 113-126. doi: 10.1016/S1474-4422(13)70233-3

Dalla Costa, G., Martinelli, V., Moiola, L., Sangalli, F., Colombo, B., Finardi, A., et al. (2019). Serum neurofilaments increase at progressive multifocal leukoencephalopathy onset in natalizumab-treated multiple sclerosis patients. Ann. Neurol. 85, 606-610. doi: 10.1002/ana.25437

Delcoigne, B., Manouchehrinia, A., Barro, C., Benkert, P., Michalak, Z., Kappos, L., et al. (2020). Blood neurofilament light levels segregate treatment effects in multiple sclerosis. Neurology 94, e1201-e1212. doi: 10.1212/WNL. 0000000000009097

Disanto, G., Adiutori, R., Dobson, R., Martinelli, V., Costa, G. D., Runia, T., et al. (2016). Serum neurofilament light chain levels are increased in patients with a clinically isolated syndrome. J. Neurol. Neurosurg. Psychiatry 87, 126-129. doi: 10.1136/jnnp-2014-309690

Disanto, G., Barro, C., Benkert, P., Naegelin, Y., Schädelin, S., Giardiello, A., et al. (2017). Serum Neurofilament light: a biomarker of neuronal damage in multiple sclerosis. Ann. Neurol. 81, 857-870. doi: 10.1002/ana.24954

Eikelenboom, M. J., Petzold, A., Lazeron, R. H. C., Silber, E., Sharief, M., Thompson, E. J., et al. (2003). Multiple sclerosis: neurofilament light chain antibodies are correlated to cerebral atrophy. Neurology 60, 219-223. doi: 10. 1212/01.WNL.0000041496.58127.E3

Engel, S., Steffen, F., Uphaus, T., Scholz-Kreisel, P., Zipp, F., Bittner, S., et al. (2020). Association of intrathecal pleocytosis and IgG synthesis with axonal damage in early MS. Neurol. Neuroimmunol. neuroinflammation 7, 1-10. doi: 10.1212/NXI.0000000000000679

Evered, L., Silbert, B., Scott, D. A., Zetterberg, H., and Blennow, K. (2018). Association of changes in plasma neurofilament light and tau levels with anesthesia and surgery. JAMA Neurol. 75, 542-547. doi: 10.1001/jamaneurol. 2017.4913

Forgrave, L. M., Ma, M., Best, J. R., and DeMarco, M. (2019). The diagnostic performance of neurofilament light chain in CSF and blood for Alzheimer's disease, frontotemporal dementia, and amyotrophic lateral sclerosis: a systematic review and meta-analysis. Alzheimers Dement. Diagn. Assess. Dis. Monit. 11, 730-743.

Freedman, M. S., Devonshire, V., Duquette, P., Giacomini, P. S., Giuliani, F., Levin, M. C., et al. (2020). Treatment optimization in multiple sclerosis: canadian MS working group recommendations. Can. J. Neurol. Sci. 47, 437-455. doi: $10.1017 / \operatorname{cjn} .2020 .66$ 
Fuchs, E., and Cleveland, D. W. (1998). A structural scaffolding of intermediate filaments in health and disease. Science 279, 514-519. doi: 10.1126/science.279. 5350.514

Giovannoni, G. (2018). Disease-modifying treatments for early and advanced multiple sclerosis. Curr. Opin. Neurol. 31, 233-243. doi: 10.1097/WCO. 0000000000000561

Hendricks, R., Baker, D., Brumm, J., Davancaze, T., Harp, C., Herman, A., et al. (2019). Establishment of neurofilament light chain Simoa assay in cerebrospinal fluid and blood. Bioanalysis 11, 1405-1418. doi: 10.4155/bio-2019-0163

Huss, A., Senel, M., Abdelhak, A., Mayer, B., Kassubek, J., Ludolph, A. C., et al. (2020). Longitudinal serum neurofilament levels of multiple sclerosis patients before and after treatment with first-line immunomodulatory therapies. Biomedicines 8, 1-12. doi: 10.3390/biomedicines8090312

Hviid, C. V. B., Knudsen, C. S., and Parkner, T. (2020). Reference interval and preanalytical properties of serum neurofilament light chain in Scandinavian adults. Scand. J. Clin. Lab. Invest. 80, 291-295. doi: 10.1080/00365513.2020. 1730434

Javed, A., and Stankiewicz, J. (2020). Point/Counterpoint: neurofilament light ready for prime time as a biomarker for multiple sclerosis or not? Practical Neurology, 5420. Available online at: https://practicalneurology.com/articles/ 2020-feb/pointcounterpoint-neurofilament-light

Kalm, M., Boström, M., Sandelius, Å, Eriksson, Y., Ek, C. J., Blennow, K., et al. (2017). Serum concentrations of the axonal injury marker neurofilament light protein are not influenced by blood-brain barrier permeability. Brain Res. 1668, 12-19. doi: 10.1016/j.brainres.2017.05.011

Khalil, M., Pirpamer, L., Hofer, E., Voortman, M. M., Barro, C., Leppert, D., et al. (2020). Serum neurofilament light levels in normal aging and their association with morphologic brain changes. Nat. Commun. 11, 1-9. doi: 10.1038/s41467020-14612-6

Khalil, M., Teunissen, C. E., Otto, M., Piehl, F., Sormani, M. P., Gattringer, T., et al. (2018). Neurofilaments as biomarkers in neurological disorders. Nat. Rev. Neurol. 14, 577-589. doi: 10.1038/s41582-018-0058-z

Korley, F. K., Goldstick, J., Mastali, M., Van Eyk, J. E., Barsan, W., Meurer, W. J., et al. (2019). Serum NfL (neurofilament light chain) levels and incident stroke in adults with diabetes mellitus. Stroke 50, 1669-1675. doi: 10.1161/STROKEAHA. 119.024941

Kuhle, J., Barro, C., Andreasson, U., Derfuss, T., Lindberg, R., Sandelius, Å, et al. (2016a). Comparison of three analytical platforms for quantification of the neurofilament light chain in blood samples: ELISA, electrochemiluminescence immunoassay and Simoa. Clin. Chem. Lab. Med. 54, 1655-1661. doi: 10.1515/ cclm-2015-1195

Kuhle, J., Barro, C., Disanto, G., Mathias, A., Soneson, C., Bonnier, G., et al. (2016b). Serum neurofilament light chain in early relapsing remitting MS is increased and correlates with CSF levels and with MRI measures of disease severity. Mult. Scler. 22, 1550-1559. doi: 10.1177/1352458515623365

Kuhle, J., Barro, C., Hrusovsky, K., Chang, K., Jeromin, A., Bridel, C., et al. (2018). International multi-site analytical validation of the Simoa NF-light assay in human serum samples from multiple sclerosis patients. ECTRIMS Online Libr. 228383:539.

Leppert, D., and Kuhle, J. (2019). Blood neurofilament light chain at the doorstep of clinical application. Neurol. Neuroimmunol. NeuroInflammation 6, 4-5. doi: 10.1212/NXI.0000000000000599

Lorscheider, J., and Benkert, P. (2020). Serum neurofilament light chain captures and predicts confirmed progression independent of relapses (PIRA) in multiple sclerosis. MS Virtual [Epub ahead of print].

Lublin, F. D. (2012). Disease activity free status in MS. Mult. Scler. Relat. Disord. 1, 6-7. doi: 10.1016/j.msard.2011.08.001

Manouchehrinia, A., Piehl, F., Hillert, J., Kuhle, J., Alfredsson, L., Olsson, T., et al. (2020). Confounding effect of blood volume and body mass index on blood neurofilament light chain levels. Ann. Clin. Transl. Neurol. 7, 139-143. doi: $10.1002 / \operatorname{acn} 3.50972$

Nielsen, H. H., Soares, C. B., Høgedal, S. S., Madsen, J. S., Hansen, R. B., Christensen, A. A., et al. (2020). Acute neurofilament light chain plasma levels correlate with stroke severity and clinical outcome in ischemic stroke patients. Front. Neurol. 11:448. doi: 10.3389/fneur.2020.00448

Rissin, D. M., Kan, C. W., Campbell, T. G., Howes, S. C., Fournier, D. R., Song, L., et al. (2010). Single-molecule enzyme-linked immunosorbent assay detects serum proteins at subfemtomolar concentrations. Nat. Biotechnol. 28, 595-599. doi: $10.1038 /$ nbt.1641
Rosso, M., Gonzalez, C. T., Healy, B. C., Saxena, S., Paul, A., Bjornevik, K., et al. (2020). Temporal association of sNfL and gad-enhancing lesions in multiple sclerosis. Ann. Clin. Transl. Neurol. 7, 945-955. doi: 10.1002/acn3.51060

Sartori, A., Abdoli, M., and Freedman, M. S. (2017). Can we predict benign multiple sclerosis? Results of a 20-year long-term follow-up study. J. Neurol. 264, 1068-1075. doi: 10.1007/s00415-017-8487-y

Sejbaek, T., Nielsen, H. H., Penner, N., Plavina, T., Mendoza, J. P., Martin, N. A., et al. (2019). Dimethyl fumarate decreases neurofilament light chain in CSF and blood of treatment naïve relapsing MS patients. J. Neurol. Neurosurg. Psychiatry 90, 1324-1330. doi: 10.1136/jnnp-2019-321321

Shahim, P., Politis, A., van der Merwe, A., Moore, B., Chou, Y. Y., Pham, D. L., et al. (2020). Neurofilament light as a biomarker in traumatic brain injury. Neurology 95, e610-e622. doi: 10.1212/WNL.0000000000009983

Shahim, P., Tegner, Y., Marklund, N., Blennow, K., and Zetterberg, H. (2018). Neurofilament light and tau as blood biomarkers for sportsrelated concussion. Neurology 90, E1780-E1788. doi: 10.1212/WNL.00000000 00005518

Silber, E., Semra, Y. K., Gregson, N. A., and Sharief, M. K. (2002). Patients with progressive multiple sclerosis have elevated antibodies to neurofilament subunit. Neurology 58, 1372-1381. doi: 10.1212/WNL.58.9.1372

Siller, N., Kuhle, J., Muthuraman, M., Barro, C., Uphaus, T., Groppa, S., et al. (2018). Serum neurofilament light chain is a biomarker of acute and chronic neuronal damage in early multiple sclerosis. Mult. Scler. J. 25, 678-686. doi: $10.1177 / 1352458518765666$

Thebault, S., Abdoli, M., Fereshtehnejad, S. M., Tessier, D., Tabard-Cossa, V., and Freedman, M. S. (2020a). Serum neurofilament light chain predicts long term clinical outcomes in multiple sclerosis. Sci. Rep. 10, 1-11. doi: 10.1038/s41598020-67504-6

Thebault, S., Booth, R. A., and Freedman, M. S. (2020b). Blood neurofilament light chain: the neurologist' s troponin?. Biomedicines 8:523.

Thebault, S., Bose, G., Booth, R., and Freedman, M. S. (2021). Serum neurofilament light in MS: the first true blood-based biomarker?. Mult. Scler. J. [Epub ahead of print]. doi: 10.1177/1352458521993066

Thebault, S., Lee, H., Bose, G., Tessier, D., Abdoli, M., Bowman, M., et al. (2020c). Neurotoxicity after hematopoietic stem cell transplant in multiple sclerosis. Ann. Clin. Transl. Neurol. 7, 767-775. doi: 10.1002/acn3.51045

Thebault, S., Tessier, D., Lee, H., Bowman, M., Bar-Or, A., Arnold, D. L., et al. (2019). High serum neurofilament light chain normalises after haematopoietic stem cell transplant for MS. Neurol. Neuroimmunol. Neuroinflammation 6:e598. doi: 10.1212/NXI.0000000000000598

Uher, T., McComb, M., Galkin, S., Srpova, B., Oechtering, J., Barro, C., et al. (2020a). Neurofilament levels are associated with blood-brain barrier integrity, lymphocyte extravasation, and risk factors following the first demyelinating event in multiple sclerosis. Mult. Scler. J. 27, 220-231. doi: 10.1177/ 1352458520912379

Uher, T., Schaedelin, S., Srpova, B., Barro, C., Bergsland, N., Dwyer, M., et al. (2020b). Monitoring of radiologic disease activity by serum neurofilaments in MS. Neurol. Neuroimmunol. Neuroinflammation 7:714. doi: 10.1212/NXI. 0000000000000714

Wilson, D. H., Rissin, D. M., Kan, C. W., Fournier, D. R., Piech, T., Campbell, T. G., et al. (2016). The simoa HD-1 analyzer: a novel fully automated digital immunoassay analyzer with single-molecule sensitivity and multiplexing. J. Lab. Autom. 21, 533-547. doi: 10.1177/2211068215589580

Yaldizli, Ö, Benkert, P., Maceski, A., Barakovic, M., Todea, R., Cagol, A., et al. (2020). Value of serum neurofilament light chain levels as a biomarker of suboptimal treatment response in MS clinical practice. MS Virtual [Epub ahead of print].

Conflict of Interest: The authors declare that the research was conducted in the absence of any commercial or financial relationships that could be construed as a potential conflict of interest.

Copyright (c) 2021 Thebault, Booth, Rush, Maclean and Freedman. This is an openaccess article distributed under the terms of the Creative Commons Attribution License (CC BY). The use, distribution or reproduction in other forums is permitted, provided the original author(s) and the copyright owner(s) are credited and that the original publication in this journal is cited, in accordance with accepted academic practice. No use, distribution or reproduction is permitted which does not comply with these terms. 